\title{
THE NONABELIAN TENSOR SQUARE OF A FINITE SPLIT METACYCLIC GROUP
}

\author{
by D. L. JOHNSON*
}

(Received 10th August 1985)

Given any group $G$, its tensor square $G \otimes G$ is defined by the following presentation (see [3]):

$$
\begin{aligned}
\text { generators: } & g \otimes h, \\
& g g^{\prime} \otimes h=\left({ }^{g} g^{\prime} \otimes{ }^{g} h\right)(g \otimes h), \\
\text { relations: } & g \otimes h h^{\prime}=(g \otimes h)\left({ }^{h} g \otimes{ }^{h} h^{\prime}\right)
\end{aligned}
$$

where $g, g^{\prime}, h, h^{\prime}$ range independently over $G$, and ${ }^{g} h=g h g^{-1}$. In what follows, ${ }^{g} \mathbf{g}^{\prime} \otimes{ }^{g} h$ is often written in the abbreviated form ${ }^{g}\left(g^{\prime} \otimes h\right)$.

Among the groups $G$ for which $G \otimes G$ is computed in [2] is the metacyclic group

$$
G=\left\langle x, y \mid y^{n}=e, x^{m}=e, x y x^{-1}=y^{l}\right\rangle, l^{m} \equiv 1(\bmod n)
$$

in the favourable special case when $n$ is odd. It is the direct product of four cyclic groups, of orders $m,(n, l-1),\left(n, l-1,1+l+\cdots+l^{m-1}\right)$, and $\left(n, 1+l+\cdots+l^{m-1}\right)$, respectively. Our purpose here is to remedy this deficiency by evaluating $G \otimes G$ for even $n$. The following preliminary results, valid for all $G$, are stated in [3] and some proofs are given in [2].

The mapping

$$
\begin{aligned}
\kappa: G \otimes G \rightarrow G^{\prime} \\
g \otimes h \mapsto[g, h]=g h g^{-1} h^{-1}
\end{aligned}
$$

is an epimorphism. Its kernel is denoted by $J_{2}(G) ; J_{2}(G)$ is $G$-trivial and lies in the centre of $G \otimes G$.

It follows from (1) that, for all $g ; g^{\prime}, h, h^{\prime} \in G$,

$$
\left[g \otimes h, g^{\prime} \otimes h^{\prime}\right]=[g, h] \otimes\left[g^{\prime}, h^{\prime}\right] .
$$

*This paper forms part of the Proceedings of the conference Groups-St Andrews 1985. 
The mapping

$$
\begin{gathered}
\tau: G \otimes G \rightarrow G \otimes G \\
g \otimes h \mapsto(h \otimes g)^{-i}
\end{gathered}
$$

is an automorphism.

There is an exact sequence

$$
H_{3}(G) \rightarrow \Gamma G^{a b} \stackrel{\psi}{\rightarrow} J_{2}(G) \rightarrow H_{2}(G) \rightarrow 0
$$

where $\Gamma$ is Whitehead's quadratic functor (see [4]), and $\operatorname{Im} \psi$ is generated by the elements $g \otimes g, g \in G$.

We assume henceforth that $G$ is the metacyclic group given by (2). The calculation now proceeds in a number of steps.

(7). We first note two consequences of (3). Being an extension of (the central) $J_{2}(G)$ by (the cyclic) $G^{\prime}, G \otimes G$ is abelian. Secondly, $x$ and $y$ fix each of $x \otimes x, y \otimes y$, $(x \otimes y)(y \otimes x)$. It is clear from (1) that these three elements, together with $x \otimes y$, generate $G \otimes G$ qua $G$-module. Our first main aim is to show that they generate $G \otimes G$ as a group.

$$
(y \otimes y)^{n}=e=a^{2}, \text { where } a=(y \otimes y)^{l-1} .
$$

First, it follows from (4) and (1) that

$$
(y \otimes y)^{(l-1)^{2}}=y^{l-1} \otimes y^{l-1}=[x, y] \otimes[x, y]=[x \otimes y, x \otimes y]=e
$$

Next, by (7) and (2),

$$
y \otimes y={ }^{x}(y \otimes y)=y^{l} \otimes y^{l}=(y \otimes y)^{l^{2}}
$$

Finally, (1) implies that

$$
(y \otimes y)^{n}=y \otimes y^{n}=y \otimes e=e .
$$

These three equations together yield (8). Substitution of $e$ for $a$ in what follows gives a replica of the calculation in [2] for $n$ odd.

$$
y(x \otimes y)=(x \otimes y) a
$$

Using (2), (1) and (8),

$$
\begin{aligned}
y(x \otimes y) & =\left({ }^{y} x\right) \otimes y=\left(y^{1-l} x\right) \otimes y=y^{y^{1-1}}(x \otimes y)\left(y^{1-l} \otimes y\right) \\
& =y^{1-1}(x \otimes y)(y \otimes y)^{1-l}=y^{y^{1-1}}(x \otimes y) a .
\end{aligned}
$$


Thus $y^{l}$ acts on $x \otimes y$ as multiplication by $a$. Hence the action of $y=y^{l m}=\left(y^{l}\right)^{l^{m-1}}$ multiplies $x \otimes y$ by $a^{i^{m-1}}$. Since $n$ is even, (2) implies that $l$ is odd, and the result follows from (8).

$$
{ }^{x}(x \otimes y)=(x \otimes y)^{l} a^{(l-1) / 2} .
$$

Using (9) and the second relation of (1) $l-1$ times each, together with (8) and the centrality of $a(7)$,

$$
\begin{aligned}
x(x \otimes y) & =x \otimes x^{x} y=x \otimes y^{l} \\
& =(x \otimes y)^{y}\left(x \otimes y^{l-1}\right)=\cdots \\
& =\prod_{k=0}^{l-1} y^{k}(x \otimes y)=(x \otimes y)^{l} a^{(l-1) / 2} .
\end{aligned}
$$

This achieves our first objective: $G \otimes G$ is four-generated.

$$
x^{p} \otimes y^{q}=(x \otimes y)^{q \cdot p} a^{r}, y^{q} \otimes x^{p}=(y \otimes x)^{q \cdot p} a^{r},
$$

where

$$
r=(p q(q-2)+q \cdot p) / 2 \text { and } q \cdot p=q\left(1+l+\cdots+l^{p-1}\right) .
$$

Repeated use of the relations (1) gives

$$
\begin{aligned}
x^{p} \otimes y^{q} & ={ }^{x}\left(x^{p-1} \otimes y^{q}\right)\left(x \otimes y^{q}\right) \\
& =\prod_{k=p-1}^{0} x^{k}\left(x \otimes y^{q}\right)
\end{aligned}
$$

and

$$
x \otimes y^{q}=\prod_{k=0}^{q-1} y^{y^{k}}(x \otimes y)=(x \otimes y)^{q} a^{q(q-1) / 2}
$$

by (7). Using (10),

$$
x^{p} \otimes y^{q}=\prod_{k=p-1}^{0} x^{k}(x \otimes y)^{q} a^{q(q-1) / 2}=(x \otimes y)^{q \cdot p} a^{r},
$$

where

$$
r=p q(q-1) / 2+\sum_{k=p-1}^{0} q\left(l^{k}-1\right) / 2=p q(q-1) / 2+(q \cdot p-p q) / 2,
$$

as claimed. The second formula now follows by applying the automorphism $\tau$ of (5) to 
the first.

$$
y^{p} x^{q} \otimes y^{r} x^{s}=(x \otimes x)^{q s}(y \otimes y)^{p r}(x \otimes y)^{r \cdot q}(y \otimes x)^{p \cdot s} a^{t}
$$

where

$$
t=p r(q+s)+(1 / 2)(q r(r-2)+s p(p-2)+r . q+p . s)
$$

Using (1),

$$
\begin{aligned}
y^{p} x^{q} \otimes y^{r} x^{s} & =y^{p}\left(x^{q} \otimes y^{r} x^{s}\right)\left(y^{p} \otimes y^{r} x^{s}\right) \\
& =y^{p}\left(x^{q} \otimes y^{r}\right)^{y^{p+r}}\left(x^{q} \otimes x^{s}\right)\left(y^{p} \otimes y^{r}\right)^{y^{r}}\left(y^{p} \otimes x^{s}\right),
\end{aligned}
$$

and the result follows from $(11),(9)$ and $\tau(9)$, since $p(r \cdot q)+r(p \cdot s) \equiv \operatorname{pr}(q+s)(\bmod 2)$.

$$
\begin{aligned}
& (y \otimes y)^{n}=e=(x \otimes x)^{m}, \\
& (y \otimes x)^{n}=e=(x \otimes y)^{n} \\
& (y \otimes x)^{1+l+\cdots+l^{m-1}}=\dot{a}^{(l-1) m(m-1) / 4}=(x \otimes y)^{1+l+\cdots+l^{m-1}}
\end{aligned}
$$

These are immediate consequences of the fact that the right-hand side of (12) must be independent of the choices of $p$ and $r \bmod n$ and of $q$ and $s \bmod m$.

$$
(x \otimes y)^{l-1}(y \otimes x)^{l-1}=e, a^{m}=e .
$$

Because of (3),

$$
\begin{aligned}
x \otimes x & ={ }^{y}(x \otimes x)={ }^{y} x \otimes{ }^{y} x=y^{1-l} x \otimes y^{1-l} x \\
& ={ }^{y^{1-1}}\left(x \otimes y^{1-l}\right)^{y^{2(1-l)}}(x \otimes x)\left(y^{1-l} \otimes y^{1-l}\right)^{y^{1-l}}\left(y^{1-l} \otimes x\right) \\
& =y^{y^{1-1}}\left(\left(x \otimes y^{1-l}\right)\left(y^{1-l} \otimes x\right)\right)(x \otimes x)(y \otimes y)^{(1-l)^{2}},
\end{aligned}
$$

so that

$$
\left(x \otimes y^{1-l}\right)\left(y^{1-l} \otimes x\right)=e
$$

using (8). The first relation now follows from (11). For the second, $y$ fixes $(y \otimes x)^{1+l+\cdots+l^{m-1}}$ by (13), but (by $\left.\tau(9)\right)$ multiplies it by $a^{1+l+\cdots+l^{m-1}}=a^{m}$, since $l$ is odd and $a^{2}=e$.

(15). The relations (8), (13) and (14) now define $G \otimes G$ as an abelian group on the generators $y \otimes y, x \otimes x, x \otimes y$ and $y \otimes x$. For, let $Y=y \otimes y, X=x \otimes x, T=x \otimes y$, $Z=(x \otimes y)(y \otimes x)$, and retain the abbreviation $A=a$ for convenience. Then these 
relations are equivalent to the following:

$$
\begin{aligned}
& Y^{n}=e, Y^{l-1}=A, A^{2}=e=A^{m}, X^{m}=e, \\
& T^{n}=e, T^{1+l+\cdots+l^{m-1}}=A^{(l-1)(m-1) m / 4}, \\
& Z^{l-1}=Z^{n}=Z^{1+l+\cdots+l^{m-1}}=e .
\end{aligned}
$$

On the other hand, the mapping

$$
\gamma: y^{p} x^{p} \otimes y^{r} x^{s} \mapsto X^{q s} Y^{p r} Z^{p . s} T^{r . q-p . s} A^{p r(q+s)+(1 / 2)(q r(r-2)+s p(p-2)+r . q+p . s)}
$$

preserves the relations (1), by tedious and omitted checking. (Note that, since $\gamma \tau=\tau \gamma$ on the generators, we only need to check one of the two relations).

(16) Proposition. If $G$ is the metacyclic group given by (2), then $G \otimes G$ is the abelian group with generators $T, X, Y, Z, A$ and relations (15). Furthermore, $M(G)$ is cyclic of order $(n, l-1)\left(n, 1+l+\cdots+l^{m-1}\right) / n$, (cf. [1]).

Proof. The first assertion is proved above. For the second, note that $M(G)$ is cyclic since $G$ has deficiency $\geqq-1$, and its order is given by (6) as

$$
\left|J_{2}(G): \operatorname{Im} \psi\right|=\frac{|G \otimes G|}{\left|G^{\prime}\right|\langle g \otimes g, g \in G\rangle},
$$

by (3). By (12), $\operatorname{Im} \psi=\langle g \otimes g, g \in G\rangle=\langle X, Y, Z\rangle$. By (15), $\langle X, Y, Z\rangle$ has index (n, $\left.1+l+\cdots+l^{m-1}\right)$ in $G \otimes G$, and $G^{\prime}$ has index $m(n, l-1)$ in $G$ from (2).

\section{REFERENCES}

1. R. BeYL, The Schur multiplicator of metacyclic groups, Proc. Amer. Math. Soc. 40 (1973), 413-418.

2. R. Brown, D. L. Johnson and E. F. Robertson, Some computations of non-abelian tensor products of groups, J. Algebra, to appear.

3. R. Brown and J.-L. Loday, Excision homotopique en basse dimension, C.R. Acad. Sci. Paris Sér. I Math. 298: 15 (1984), 353-356.

4. J. H. C. WhiteheAd, A certain exact sequence, Ann. of Math. 52 (1950), 51-110.

Department of Mathematics

UNIVERSITY OF NOTTINGHAM

University Park

NOTTINGHAM NG7 2RD 